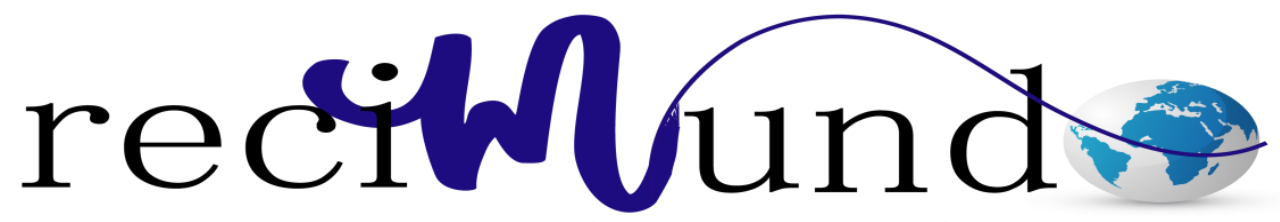

Revista Cientifica Mundo de la Investigación y el Conocimiento

Walter Alejandro Patiño Zambrano ${ }^{\text {a; }}$ José Eduardo Cedeño Gilces ${ }^{\text {b; }}$ María Alexandra Sánchez Vélez ${ }^{\mathrm{c}}$; Steven Javier Berruz Alvarado ${ }^{\mathrm{d}}$

Autoimagen corporal y procedimientos de cirugía plástica estética reconstructiva

Revista Científica Mundo de la Investigación y el Conocimiento. Vol. 2 núm., 1, febrero, ISSN: 2588-073X, 2018, pp. 658-673

DOI: 10.26820/recimundo/2.1.2018.658-673

Editorial Saberes del Conocimiento

Recibido: 05/12/2017 Aceptado: 10/02/2018
a. alej08585@hotmail.com
b. Médico; Universidad de Guayaquil; educedenomd @ hotmail.com
c. Médico, Universidad de Guayaquil; marialexa.-.1984@ hotmail.com
d. Noveno Semestre de la Carrera de Medicina; Steven_berruz@hotmail.com 


\section{Autoimagen corporal y procedimientos de cirugía plástica estética \\ reconstructiva}

Vol. 2, núm. 1., (2018)

Walter Alejandro Patiño Zambrano; José Eduardo Cedeño Gilces; María Alexandra Sánchez

Vélez; Steven Javier Berruz Alvarado

\section{RESUMEN}

La cirugía reconstructiva es una de las grandes áreas de la cirugía plástica, debido a que su objetivo es reparar dificultades o afecciones de salud en el cuerpo, pese a que se diferencia de la cirugía estética, la cirugía reconstructiva conlleva un buen porcentaje de las actividades de los cirujanos plásticos con una connotación muy seria, ya que en algunos casos pueden tratarse complicaciones que ponen en riesgo la vida o la calidad de vida de un paciente.

Sus aplicaciones dependerán del caso específico de cada paciente para conocer el alcance y la metodología a elegir por el cirujano. Sin embargo, los procedimientos más habituales a corregir son: Secuelas de procedimientos de oncología, traumas maxilofaciales, quemaduras, cirugía de las manos, malformaciones congénitas. Aspectos estos a ser abordados en el presente artículo.

Palabras claves: Autoimagen, procedimientos quirúrgicos, cirugía estética, cirugía reconstructiva. 


\section{Autoimagen corporal y procedimientos de cirugía plástica estética reconstructiva}

Vol. 2, núm. 1., (2018)

Walter Alejandro Patiño Zambrano; José Eduardo Cedeño Gilces; María Alexandra Sánchez Vélez; Steven Javier Berruz Alvarado

Reconstructive surgery is one of the great areas of plastic surgery, because its goal is to repair difficulties or health conditions in the body, although it differs from cosmetic surgery, reconstructive surgery involves a good percentage of activities of plastic surgeons with a very serious connotation, since in some cases complications can be treated that endanger the life or quality of life of a patient.

Its applications will depend on the specific case of each patient to know the scope and methodology to be chosen by the surgeon. However, the most common procedures to correct are: Sequelae of oncology procedures, maxillofacial traumas, burns, hand surgery, congenital malformations. Aspects these to be addressed in this article.

Keywords: Self-image, surgical procedures, aesthetic surgery, reconstructive surgery. 


\section{Autoimagen corporal y procedimientos de cirugía plástica estética \\ reconstructiva}

Vol. 2, núm. 1., (2018)

Walter Alejandro Patiño Zambrano; José Eduardo Cedeño Gilces; María Alexandra Sánchez

Vélez; Steven Javier Berruz Alvarado

\section{Introducción.}

El fenómeno de la distorsión en la autoimagen corporal tiene en la actualidad varias explicaciones claras pero complejas en la psicología cognitivo-conductual y en la neurofisiología. Existen numerosas teorías que explican este fenómeno. Aunado al mensaje cultural que sostiene que la esbeltez es preferible, existen periodos particulares como la pubertad en los cuales los cambios en la complexión corporal engendran confusión, provocando el que con mayor facilidad se presenten distorsiones en la auto-percepción corporal (Gimenez, Correché, \& Rivarola, 2013).

A partir de una necesidad de satisfacción y/o mejoramiento de la percepción del propio ser de un paciente que ha sido abatido emocionalmente producto de haber padecido una enfermedad terminal, surge la cirugía estética y reconstructiva para dar respuesta a pacientes que requerían recuperar la funcionabilidad de la parte del cuerpo afectada o bien de una persona que ha nacido con un defecto congénito.

Es por ello que a lo largo de este estudio bibliográfico se aborda el tema de la autoimagen y los procedimientos más comunes en materia de cirugías plásticas y reconstructivas, cuyo análisis se basó en textos y en visiones de expertos, para así tratar de dar un enfoque más actualizado en términos de lo que implica en el siglo XXI la autoimagen y la cirugía médica reconstructiva, la cual ha evolucionado para incluir dentro de los procedimientos aplicados el aspecto de estética. 


\section{Autoimagen corporal y procedimientos de cirugía plástica estética reconstructiva}

Vol. 2, núm. 1., (2018)

Walter Alejandro Patiño Zambrano; José Eduardo Cedeño Gilces; María Alexandra Sánchez Vélez; Steven Javier Berruz Alvarado

\section{Materiales y métodos}

\section{Definiciones teóricas}

Autoimagen: La autoimagen es el conjunto de creencias que se tiene sobre el cuerpo, a su vez representa la serie de emociones que siente un ser humano sobre su propio cuerpo y la percepción de este en el espacio, la cual va cambiando a lo largo de la vida de cada individuo a través del desarrollo corporal, debido a los cambios físicos (la pubertad, la adolescencia y la vejez) etapas estas por las cuales atraviesa el ser humano, periodos donde más les cuesta adaptarse a su nueva autoimagen, finalmente es importante destacar que la autoimagen corporal influye en la vida social y en la conducta de un ser en todos sus ámbitos (Vaquero-Cristóbal, Alacid, \& Muyor, 2013).

Cirugía plástica: De acuerdo a (Sánchez Rodríguez \& Alessandrini González, 2007):

La cirugía plástica es la especialidad médica que tiene por objeto la corrección y mejoramiento de anormalidades de origen congénito, adquirido, tumoral o involutiva que requieran reparación o reposición de la forma corporal y su función, mediante la cirugía se da tratamiento para reconstruir las deformidades y proporcionar función transportando tejidos del mismo cuerpo con múltiples técnicas, el uso de implantes de materiales biocomplatibles ya sea para soporte o función (movimiento), o la combinación de los dos; y en época más reciente con el uso de unidades de tejidos completo de donantes como lo son la mano y la cubierta de la cara.

La finalidad es que el paciente que ha nacido con un defecto congénito, o que ha sufrido un accidente con pérdida de la función alcance la mayor normalidad tanto en su aspecto como en 


\section{Autoimagen corporal y procedimientos de cirugía plástica estética \\ reconstructiva}

Vol. 2, núm. 1., (2018)

Walter Alejandro Patiño Zambrano; José Eduardo Cedeño Gilces; María Alexandra Sánchez

Vélez; Steven Javier Berruz Alvarado

la función de su cuerpo. También, se incluye a todos aquellos individuos sanos y emocionalmente estables que desean cambiar su aspecto ante la inconformidad con su apariencia. En este sentido, con el desarrollo de la cirugía plástica se aprendió que toda cirugía debe incluir en su diseño una gran parte estética y lo más cercana posible a la forma natural normal, dejando en la historia los procedimientos que resolvían la función, pero no eran estéticos. Es un objetivo diseñar de forma estética al remodelar los cuerpos para mejorarlos cualquiera sea la causa de la cirugía o al hacerlos más "hermosos". La cirugía plástica se ha dividido desde un punto de vista práctico en dos campos de acción.

a) Cirugía reconstructiva o reparadora, que incluye a la microcirugía, está enfocada en disimular y reconstruir los efectos destructivos de un accidente o trauma, los defectos de una malformación congénita y de una resección tumoral u oncológica. La cirugía reconstructiva o reparadora hace uso de distintas técnicas: de osteosíntesis, de traslado de tejidos mediante colgajos y trasplantes autólogos de partes del cuerpo sanas a las afectadas, etc.

b) Cirugía estética o cosmética, es realizada con la finalidad de modificar aquellas partes del cuerpo que no son satisfactorias para el paciente. En muchos casos, sin embargo, hay razones médicas (por ejemplo, mamoplastia de reducción cuando hay problemas ortopédicos en la columna lumbar, o la blefaroplastia en casos graves de dermatocalasia que incluso impiden la vista). 


\section{Autoimagen corporal y procedimientos de cirugía plástica estética reconstructiva}

Vol. 2, núm. 1., (2018)

Walter Alejandro Patiño Zambrano; José Eduardo Cedeño Gilces; María Alexandra Sánchez Vélez; Steven Javier Berruz Alvarado

Beneficios y Contras de la cirugía reconstructiva

Los beneficios que se obtienen de una cirugía reconstructiva son múltiples, siendo de alto impacto no solo en aspectos de funcionalidad, dándole un sentido de proporción y naturalidad a una parte del cuerpo humano, sino que coadyuva a mejorar la autoestima del paciente y por ende a la percepción que tiene de sí mismo a través de su autoimagen. Una reconstrucción perfectamente ejecutada puede devolver a la mayoría de las zonas del cuerpo una condición casi perfecta, debido a que cumple con un propósito práctico (Ortiz Monasterio \& Berlanga Ramírez, 2004), ya que las zonas del cuerpo que tienen una función importante pueden verse gravemente impedidas de realizar su actividad debido a un trauma físico o a un defecto de nacimiento.

Según (Trelles, 2013):

El ISAPS (Encuesta Internacional sobre Procedimientos Estéticos/Cosméticos) reporta que para el 2014, aproximadamente 1.48 millones de procedimientos quirúrgicos y 2.58 millones de procedimientos mínimamente invasivos, o un total combinado de 4.06 millones de procedimientos fueron realizados sólo en EE.UU. Sin duda alguna, la popularidad de la cirugía plástica se está incrementando especialmente en EE.UU. El cual se encuentra en la lista de los 8 países encuestados, incluyendo a Brasil, Japón, Corea del Sur, México, Alemania, Francia y Colombia. Es evidente que cada día 


\section{Autoimagen corporal y procedimientos de cirugía plástica estética \\ reconstructiva}

Vol. 2, núm. 1., (2018)

Walter Alejandro Patiño Zambrano; José Eduardo Cedeño Gilces; María Alexandra Sánchez

Vélez; Steven Javier Berruz Alvarado

existen una mayor tendencia por parte de los pacientes para someterse

a este tipo de intervención quirúrgica, ya que el paciente apuesta más

hacia los beneficios que se obtendrá post-tratamiento quirúrgico que a

las posibles complicaciones, el largo proceso de recuperación, posible

proceso infeccioso y los dolores postoperatorios, entre otro tipo de

inconvenientes que pudiera presentar

Ventajas:

a) No es tan doloroso: Los avances en el equipamiento médico y dispositivos quirúrgicos han hecho a la cirugía plástica y reconstructiva cada día menos invasiva y por ende menos dolorosa que lo que era en el pasado, específicamente hace dos décadas (Ortiz Monasterio \& Berlanga Ramírez, 2004).

b) Beneficiosa para la salud del paciente: Debido a que ayuda a corregir y tratar de manera inmediata un problema congénito a un paciente que no se pudiese corregir de otra manera o bien se puede tratar para mejorar función y estética de una parte del cuerpo que ha sufrido un trauma severo (quemaduras de $3^{\circ}$ grado, mastectomía debido al cáncer de mama, etc), puesto que contribuye a restaurar el balance físico y emocional del paciente (Trelles, 2013).

c) Resultados visibles de manera inmediata y en la mayoría de los casos suele ser permanente: Para mantener los efectos beneficiosos de la cirugía plástica, el paciente debe estar dispuesto a hacer cambios en su estilo de vida según las indicaciones del médico tratante (Estética C. P., n.ed). 


\section{Autoimagen corporal y procedimientos de cirugía plástica estética reconstructiva}

Vol. 2, núm. 1., (2018)

Walter Alejandro Patiño Zambrano; José Eduardo Cedeño Gilces; María Alexandra Sánchez Vélez; Steven Javier Berruz Alvarado

\section{Desventajas:}

a) Altos costos, que suelen ser prohibitivos para los pacientes al momento de tener que someterse a este tipo de intervenciones: En la mayoría de los casos, la cirugía plástica no es cubierta por ningún plan de seguro de salud y el costo varía dependiendo del procedimiento realizado (Estética C. P., n.ed). Sin embargo, se ha venido presentando en la última década una reducción en los costos en de cirugías plásticas aunado al poco temor de la gente a someterse a estos procedimientos, lo que ha incrementado el número de operaciones en los últimos años a nivel mundial. De acuerdo con datos de la Sociedad Internacional de Cirugía Plástica Estética (ISAPS, por sus siglas en inglés), este tipo de procedimientos aumentaron una media del 30\% en 2015 y en 2016, siendo Estados Unidos quien encabeza el mayor número de cirugías con 4,2 millones de operaciones estéticas (Nazar J, Zamora H, \& Vega P, 2014). El doctor Antonio Fuente del Campo, académico de la Facultad de Medicina (FM) de la Universidad Nacional Autónoma de México (UNAM), alertó que estos procedimientos se han vuelto peligrosos pues en esta área ejercen algunos médicos generales improvisados $\mathrm{y}$ otros profesionales sin especialización, lo que implica altos riesgos para el paciente, los cuales van desde no obtener los resultados esperados hasta la muerte, a su vez el especialista indicó que otro de los factores que ha favorecido el incremento en el número de cirugías es que ahora existen procedimientos endoscópicos no invasivos que permiten una recuperación más rápida y resultados con apariencia natural (Nazar J, Zamora H, \& Vega P, 2014). 


\section{Autoimagen corporal y procedimientos de cirugía plástica estética \\ reconstructiva}

Vol. 2, núm. 1., (2018)

Walter Alejandro Patiño Zambrano; José Eduardo Cedeño Gilces; María Alexandra Sánchez

Vélez; Steven Javier Berruz Alvarado

b) Una intervención quirúrgica no transforma al paciente en otra persona: La cirugía plástica no es mágica, fue creada con un propósito específico y cumple tal fin, pese a su evolución hoy día a satisfacer necesidades estéticas en su mayoría, el paciente no debe esperar tener un rostro o cuerpo completamente nuevo después de la intervención de cirugía plástica. Por ello es indispensable que se posea expectativas realistas acerca de qué puede hacer la cirugía para el paciente es el primer paso hacia una intervención exitosa.

c) Riesgos: Como cualquier intervención quirúrgica, la cirugía plástica tiene riesgos. Las complicaciones más comunes son sangrado excesivo, infección en el sitio de incisión, necrosis y reacciones adversas a la anestesia (Trelles, 2013). Cada intervención de cirugía plástica viene con su conjunto de complicaciones particulares como la pérdida de sensibilidad en el área tratada, contractura capsular en el caso de implantes, así como ruptura y filtración del implante (Estética C. P., n.ed). El edema, seroma y hematoma también son posibles complicaciones de las intervenciones de cirugía plástica que son previsivas en algunos casos, y tratables en otros (SECPRE, 2015).

Procedimientos más comunes en materia de cirugía plástica reconstructiva

La reconstrucción del seno: Para las mujeres que se han sometido a una mastectomía (Mariela Rocío, 2014). Dependiendo del problema y la gravedad del caso, existen muchos métodos diferentes para realizar una cirugía de reconstrucción de pecho. Las dos cirugías más populares son la del tejido expansor /implante de pecho y los métodos de reconstrucción del colgajo o flap (Ortiz Monasterio \& Berlanga Ramírez, 2004) 


\section{Autoimagen corporal y procedimientos de cirugía plástica estética reconstructiva}

Vol. 2, núm. 1., (2018)

Walter Alejandro Patiño Zambrano; José Eduardo Cedeño Gilces; María Alexandra Sánchez Vélez; Steven Javier Berruz Alvarado

En el método del tejido expansor se utiliza un expansor de tejido y un implante temporal que se inserta en un bolsillo situado debajo del músculo pectoral mayor del pecho de la mujer. A veces, se liberan los músculos para formar una bolsa más grande, siempre dependiendo de la dificultad del procedimiento. En este procedimiento se utiliza un injerto dérmico acelular humano o animal con la finalidad de aumentar la cobertura del implante (Ortiz Monasterio \& Berlanga Ramírez, 2004) En ese momento se inyecta de forma una solución salina para expandir el tejido superpuesto. Es un proceso que puede durar varias semanas, incluso meses. Una vez que el tejido expandido ha alcanzado el tamaño necesario, se sustituye un implante permanente por el temporal y se construyen la areola y el pezón, si fuera necesario. Este segundo paso sólo se puede llevar a cabo cuando la piel se ha estirado hasta alcanzar el tamaño previsto (Sociedad colombiana de cirugía plástica estética y reconstructiva, 2016).

La cirugía reconstructiva con colgajo o flap cuenta con un método diferente, utilizando el tejido de otras partes del cuerpo del paciente, como el tejido de las nalgas, los muslos, la espalda y el abdomen. Los colgajos de la zona donante se unen quirúrgicamente consiguiendo la forma necesaria para facilitar la reconstrucción. Por lo general, cuando la zona donante de tejidos, se encuentra cerca de la zona dañada, los vasos sanguíneos originales pueden crear un túnel bajo la piel hasta llegar a la zona del seno. Si no, se pueden crear unas nuevas conexiones sanguíneas en la zona a tratar (SECPRE, 2015).

La reducción de pecho: Para aquellas mujeres que cuentan con pechos con un volumen excesivo que les produce molestias físicas e interfiere en muchas de sus actividades diarias. El 


\section{Autoimagen corporal y procedimientos de cirugía plástica estética \\ reconstructiva}

Vol. 2, núm. 1., (2018)

Walter Alejandro Patiño Zambrano; José Eduardo Cedeño Gilces; María Alexandra Sánchez

Vélez; Steven Javier Berruz Alvarado

objetivo principal de la mamoplastia de reducción es proporcionar a la mujer unos senos más pequeños y con una forma más proporcional al resto de su cuerpo (Ortiz Monasterio \& Berlanga Ramírez, 2004).

Reconstrucción de la areola y del pezón: Se realiza cuando las mamas se han recuperado un poco después de la cirugía. El objetivo de la intervención es colocar los pezones y la areola en la posición correcta (Sociedad colombiana de cirugía plástica estética y reconstructiva, 2016). Hay varios métodos para reconstruir el pezón, el método de la composición de la areola y del pezón con injerto se puede llevar a cabo si el pezón y la areola de la zona de destino tienen un tamaño suficiente. Este método utiliza el tejido que se extrae de las zonas donantes para la reconstrucción del pezón. Se crea un colgajo de tejido para formar el pezón, éste se eleva en la zona dañada produciendo el efecto de un pezón. La areola se crea por una incisión circular y luego tanto el pezón como la areola se tatúan para que tengan el mismo color que el otro pecho (plástica, 2013).

Cirugías para pies y manos afectadas por cualquier enfermedad, incluyendo tumores (cancerígenos y no cancerígenos), dedos palmeados (cuando los dedos están unidos por una membrana), de acuerdo a (Ortiz Monasterio \& Berlanga Ramírez, 2004):

Uno de los procedimientos plásticos más importantes de la cirugía reconstructiva es la cirugía reconstructiva de manos. Debido a la importancia de las manos en la vida cotidiana, se trata de un procedimiento al que debería ser sometido cualquier persona con un defecto o lesión en la mano. La cirugía de reconstrucción de las manos no es sólo capaz de mejorar la apariencia de la mano, sino que también es capaz de mejorar su función primaria restaurando la 


\section{Autoimagen corporal y procedimientos de cirugía plástica estética reconstructiva}

Vol. 2, núm. 1., (2018)

Walter Alejandro Patiño Zambrano; José Eduardo Cedeño Gilces; María Alexandra Sánchez Vélez; Steven Javier Berruz Alvarado

funcionalidad de la mano, lo que permite al paciente vivir una vida casi completamente normal.

Dependiendo de la reparación necesaria en cada caso, hay muchos tipos de cirugía reconstructiva de mano disponibles.

La reparación del tendón es una de las cirugías más comunes en la reconstrucción de la mano. Esta cirugía trata de volver a conectar los tendones cortados o rasgados por lesiones o heridas en la mano, para restaurar el movimiento y la función de la mano.

Los dedos palmeados nos sirven como ejemplo para explicar cómo esta cirugía también puede corregir defectos de nacimiento. Dependiendo del defecto de nacimiento, el cirujano puede emplear diferentes procedimientos quirúrgicos utilizados en muchas otras cirugías reconstructivas, como por ejemplo la Z-plastia para proporcionar el doblado y la flexión de las partes de la mano que no son capaces de doblarse debido al defecto de nacimiento.

La cirugía del túnel carpiano de la mano está dirigida a las personas que sufren el síndrome del túnel carpiano, que impide el movimiento adicional de la muñeca y del mano provocado por una inflamación del nervio y de los tendones. El procedimiento quirúrgico consiste en realizar una incisión en la palma de la mano hasta la muñeca con la finalidad de liberar la presión en el túnel carpiano.

Otra de las cirugías más comunes que nos podemos encontrar es la cirugía del dedo gatillo. Esta cirugía se utiliza cuando el paciente sufre una condición física conocida como el dedo gatillo. El dedo gatillo es cuando uno de los dedos de la mano está bloqueado en una posición doblada, como si fuera a "disparar". Este bloqueo está causado por una inflamación de 


\section{Autoimagen corporal y procedimientos de cirugía plástica estética \\ reconstructiva}

Vol. 2, núm. 1., (2018)

Walter Alejandro Patiño Zambrano; José Eduardo Cedeño Gilces; María Alexandra Sánchez

Vélez; Steven Javier Berruz Alvarado

los tendones. La cirugía se realiza cuando los métodos comunes del tratamiento de la enfermedad, como los ejercicios digitales o el reposo del dedo no funcionan. Esta cirugía se lleva a cabo mediante la realización de unas pequeñas incisiones en la palma de la mano para liberar el sistema de poleas del dedo.

La extirpación de un quiste ganglionar es un proceso de drenaje, extracción y tratamiento de lo que conocemos como quistes ganglionares. Estos quistes son bultos benignos que se encuentran generalmente en la muñeca e impiden el movimiento de la mano y causan dolor.

A través de la cirugía reconstructiva de la mano pueden tratarse también muchas otras afecciones de la mano.

Cirugías faciales: Se puede corregir el labio leporino, o problemas respiratorios debido a una desviación del tabique (SECPRE, 2015).

Microcirugías reconstructivas: Consiste en el trasplante de piel, grasa, músculo o hueso a la cara con el objetivo de reconstruir cualquier estructura facial o cervical lesionada, incluyendo la mandíbula, lengua, nariz, mejilla, cuero cabelludo, etc. (Sociedad colombiana de cirugía plástica estética y reconstructiva, 2016).

\section{Conclusión.}

La imagen corporal es un aspecto esencial en la identidad personal, ya que repercute en el auto concepto y autoestima de una persona (Colás Bravo \& Castro Lemus, 2011), por lo cual la cirugía plástica y reconstructiva entra a jugar rol protagónico para mejorar la percepción de un 


\section{Autoimagen corporal y procedimientos de cirugía plástica estética reconstructiva}

Vol. 2, núm. 1., (2018)

Walter Alejandro Patiño Zambrano; José Eduardo Cedeño Gilces; María Alexandra Sánchez Vélez; Steven Javier Berruz Alvarado

paciente sobre su propia imagen, así como en mejorar aspectos de funcionalidad de una extremidad de un miembro o parte del cuerpo humano que ha sufrido un trauma severo. En este sentido, los beneficios que se generan de una cirugía reconstructiva posee un amplio radio de acción, debido principalmente en la recuperación de la funcionalidad al mismo tiempo que se propicia darle un sentido de proporción y naturalidad a una parte del cuerpo humano. Es por ello que se afirma que un procedimiento reconstructivo perfectamente ejecutado puede devolver a la mayoría de las zonas del cuerpo una condición casi perfecta, debido a que cumple con un propósito práctico (Ortiz Monasterio \& Berlanga Ramírez, 2004). A esto se suma que en la actualidad las ventajas de someterse a este tipo de procedimientos quirúrgicos es beneficiosa para la salud del paciente, por ser menos invasiva y por ende menos dolorosa y los resultados son visibles de manera inmediata (Mariela Rocío, 2014).

Sin embargo, no todo es positivo y se deben contemplar las implicaciones negativas del caso, como lo son: Altos costos, sangrado excesivo, infección en el sitio de incisión, necrosis y reacciones adversas a la anestesia. En cada intervención de cirugía plástica suele presentarse edema, seroma y hematoma (SECPRE).

\section{Bibliografía.}

Colás Bravo, M., \& Castro Lemus, N. (2011). Autoimagen corporal de los adolescentes : investigación desde una perspectiva de género. Sevilla: Universidad de Sevilla.

Estética, C. P. (n.ed). Analizando las Ventajas y Desventajas de la Cirugia Plastica. recuperado de: http://www.sociedadcirugiaplasticaperu.com/cirugia/las-ventajas-desventajas-de-lacirugia-plastica.htm.

Gimenez, P., Correché, M., \& Rivarola, M. (2013). Autoestima e Imagen Corporal. Estrategias de intervención psicológica para mejorar el bienestar psicológico en pre- adolescentes en 


\section{Autoimagen corporal y procedimientos de cirugía plástica estética \\ reconstructiva}

Vol. 2, núm. 1., (2018)

Walter Alejandro Patiño Zambrano; José Eduardo Cedeño Gilces; María Alexandra Sánchez

Vélez; Steven Javier Berruz Alvarado

una escuela de la ciudad de San Luis, Argentina. Fundamentos en Humanidades, 14(27), 83-93.

Mariela Rocío, C. (2014). Complicaciones en la Cirugia Plastica. Revista de Actualización Clínica Investiga, 49(2), 209-212.

Nazar J, C., Zamora H, M., \& Vega P, E. (2014). CIRUGÍA PLÁSTICA Y SUS COMPLICACIONES: ¿EN QUÉ DEBEMOS FIJARNOS? Revista Chilena de Cirugía, 66(6), 603-613.

Ortiz Monasterio, F., \& Berlanga Ramírez, F. (2004). VI. Cirugía plástica reconstructiva. Gaceta médica de México, 140(2), 145-150.

plástica, M. d. (2013). Procedimientos de cirugía plática. recuperado de: https://www.susmedicos.com/procedimientos.htm.

Sánchez Rodríguez, K., \& Alessandrini González, R. (2007). Algunas consideraciones éticas sobre la cirugía plástica. Revista Cubana de Cirugía, 46(4), 3-12.

SECPRE. (2015). Sociedad ecuatoriana de cirugía plástica reconstructiva y estética. Guayaquil: Cirujanos Plásticos del Guayas.

Sociedad colombiana de cirugía plástica estética y reconstructiva. (2016). Procedimientos: Cirugías plásticas estéticas. Bogota: SCCP.

Trelles, M. (2013). NUEVAS TECNOLOGÍAS EN CIRUGÍA PLÁSTICA-ESTÉTICA: Presentación. Cirugía Plástica Ibero-Latinoamericana, 39(4), 423-424.

Vaquero-Cristóbal, R., Alacid, F., \& Muyor, J. (2013). Imagen corporal; revisión bibliográfica. Nutrición Hospitalaria, 28(1), 27-35. 